\title{
Onboard/ Offboard Sensor Fusion for High-Fidelity Flapping-Wing Robot Flight Data
}

\author{
S. F. Armanini *, M. Karásek ${ }^{\dagger}$, G. C. H. E. de Croon ${ }^{\ddagger}$ and C. C. de Visser ${ }^{\S}$ \\ Delft University of Technology, Delft, 2629HS, The Netherlands
}

\section{Introduction}

Flapping-wing flyers have a remarkable performance at very low Reynolds numbers: they are highly maneuverable, potentially power efficient and versatile, typically being able to fly in a wide range of different conditions, extending from hover to fast forward flight. Hence bioinspired flapping-wing micro aerial vehicles (FWMAVs) are being researched extensively, and are expected to allow for novel applications that are not achievable with conventional fixed-wing or rotarywing aircraft. However, flapping-wing mechanisms are highly complex, due to (i) the unsteady aerodynamics, involving phenomena such as wake-capture and leading edge vortices, and (ii) the time-varying dynamics.

While significant insight is now available on both fronts, thanks to studies on insects and birds $[1,2,3,4]$, and, more recently, increasingly on robotic test platforms $[5,6,7,8,9,10$, $11,12,13,14,15]$, the high complexity of flapping flight still poses a considerable challenge to FWMAV development. Additionally, it is still considerably difficult to obtain realistic experimental data, particularly in free flight and in different flight regimes. Studies have found discrepancies between free-flight and wind tunnel data $[16,17,18]$, suggesting that wind tunnel measurements approximate free-flight data only for specific clamping positions and in some flight conditions, so that realistic insight for free flight ideally should be obtained in free-flight conditions.

For these reasons, analysis and modelling of flapping flight remain challenging, especially obtaining simple, computationally efficient models that can yield basic insight and be used for design and control in real-life applications. Hence, when flight data is obtainable, an attractive modelling option, in alternative to often complex and computationally expensive theoretical or numerical models, is system identification. Previous system identification efforts [19, 20, 21, 22, 23, 24, 25] showed that a data-driven approach can yield realistic but simple models, as well as new insight,

${ }^{*} \mathrm{PhD}$ Student, Control and Simulation Section, Faculty of Aerospace Engineering, Delft University of Technology; Kluyverweg 1, 2629HS, Delft, The Netherlands, Student Member AIAA

${ }^{\dagger}$ Postdoctoral Researcher, Control and Simulation Section, Faculty of Aerospace Engineering, Delft University of Technology; Kluyverweg 1, 2629HS, Delft, The Netherlands

${ }_{\ddagger}^{\ddagger}$ Assistant Professor, Control and Simulation Section, Faculty of Aerospace Engineering, Delft University of Technology; Kluyverweg 1, 2629HS, Delft, The Netherlands

$\S$ Assistant Professor, Control and Simulation Section, Faculty of Aerospace Engineering, Delft University of Technology; Kluyverweg 1, 2629HS, Delft, The Netherlands, Member AIAA 
for a novel and complex type of vehicle, that is not known in great depth. However relatively limited work has been done in this field so far, largely due to the difficulty of obtaining suitable data. On the other hand, many low-order dynamic and aerodynamic models currently available have not been validated with free-flight data, and particularly not in different flight regimes [26, 27, 28, 29].

Thus, a significant limitation in the analysis, modelling and design of flapping-wing vehicles, is the limited free-flight data available. Particular challenges include obtaining accurate free-flight data: (i) at the high frequencies relevant for flapping flight, (ii) in different flight regimes and (iii) during maneuvers. Such data would for instance support the development of more realistic models, possibly covering different flight regimes, a better understanding of maneuvering flight, and a better evaluation of free flight as opposed to the tethered wind tunnel case. This in turn would support improved understanding and further development of flapping-wing vehicles.

This paper proposes a sensor fusion-based method for the acquisition of accurate free-flight data for flapping-wing vehicles. Fusion of on-board inertial measurement unit (IMU) data and off-board optical tracking data is suggested as an approach to obtain high quality, more reliable measurements, that are also accurate at high frequencies and can be used for in-depth analysis of time-resolved flapping effects in free flight and during maneuvers.

External optical tracking systems [8, 30, 31], such as are often used for FWMAVs, provide accurate position measurements, but typically have a relatively low bandwidth if the intention is to consider processes happening during each flap cycle. Additionally, linear and angular velocities and accelerations have to be computed via numerical differentiation, which introduces considerable noise [16]. It can also be expected that as platforms become increasingly small, attitude data obtained from tracking alone will decrease in quality due to the limited resolution. By contrast, IMU sensors have a high bandwidth, which allows for high-frequency measurements. Accelerations and angular velocities are measured directly, however both are affected by integration drift - when used to determine attitude or velocity - and high noise levels. Hence, it is suggested that combining the approaches will yield more accurate and reliable data than either of the two approaches alone, and, in particular, data providing insight into what is happening during each flap cycle.

The forces computed from the obtained data are compared with wind tunnel measurements, to provide an initial idea of their accuracy and reliability. Additionally, implications of the suggested data processing approach for time-resolved modelling are discussed. We argue that our approach is advantageous for analysis of time-resolved aerodynamics and dynamics, giving results that are qualitatively comparable to wind tunnel ones, but more realistic since representing free flight, and providing the opportunity of considering dynamic maneuvers. The resulting data allow for the forces acting on the FWMAV in free flight (including maneuvers) to be reconstructed with high accuracy and resolution, at smaller time scales than previously considered. Meaningful information is obtained at frequencies up to five times the flapping frequency of the test vehicle, 
i.e. approximately $70 \mathrm{~Hz}$. As a comparison, previous work on a similar test platform showed that $200 \mathrm{~Hz}$-sampled optical tracking data only resulted in reliable information up to $32 \mathrm{~Hz}$ in the accelerations obtained from double differentiation [16]. The high resolution attained is relevant not only for the forces and moments, but also for the states themselves, and especially the body velocities, which cannot be estimated as effectively by either IMU or optical tracking alone. In this respect, the measurements can be considered highly valuable for analysis and system identification of FWMAVs. The suggested method is applicable to other similarly equipped FWMAV, and hence is also a valuable contribution to the FWMAV modelling community.

This paper is organized as follows. Section II introduces the test vehicle used in this study, the flight testing setup and data acquisition hardware, and explains the synchronization of different datasets. Section III discusses the data fusion process, focusing on filter design. Section IV presents the main results and briefly mentions potential uses of the collected data for time-resolved modelling and analysis. Section V contains the main conclusions. The overall approach proposed, from raw data to fused and processed data, is illustrated in fig. 1. The logic in this diagram is followed throughout the rest of this paper.

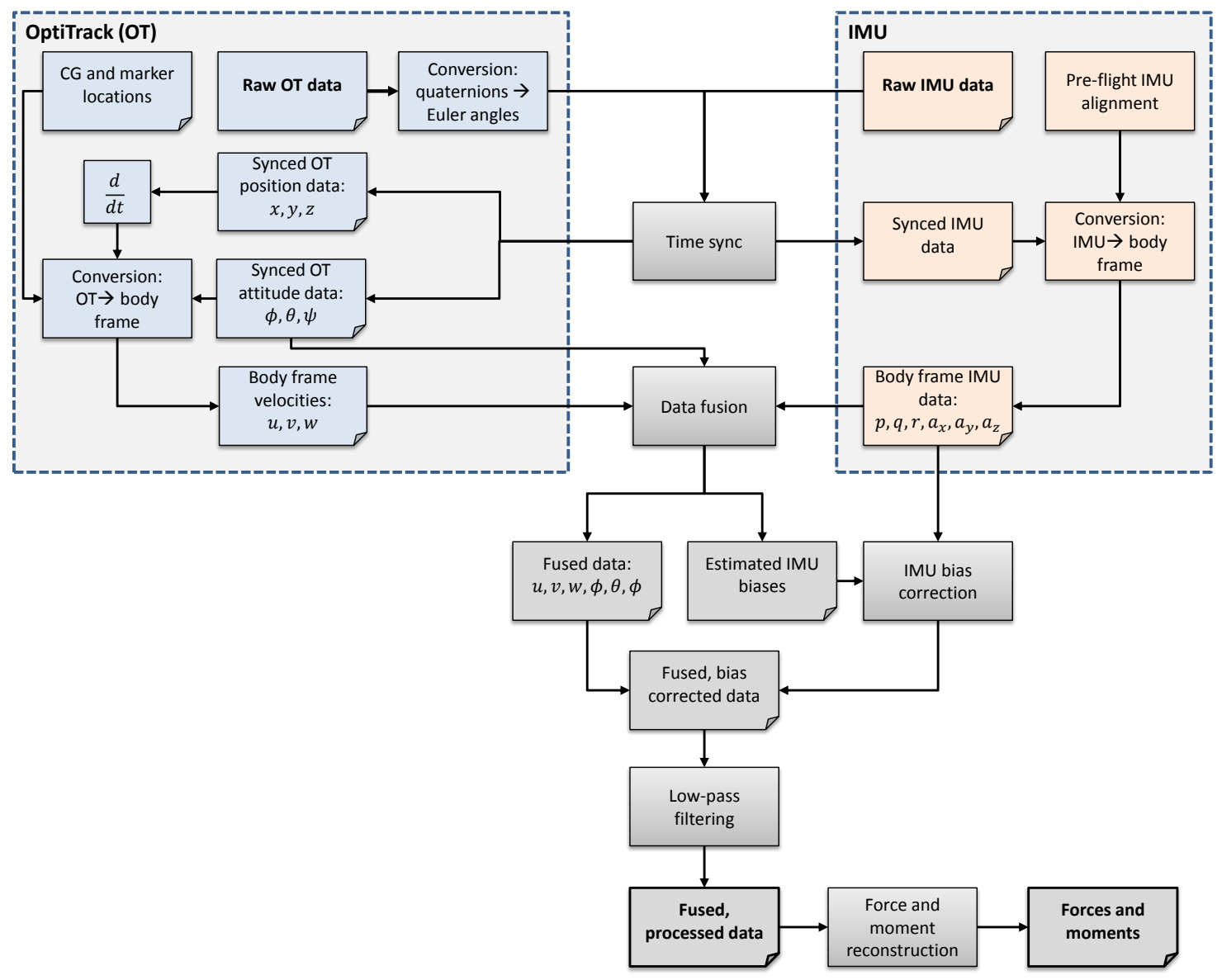

Figure 1: Diagram illustrating the proposed data processing and fusion process. 


\section{Experimental setup}

\section{A. Test vehicle}

The platform used in this study is a four-winged ornithopter (cf. fig. 2) with a $280 \mathrm{~mm}$ wingspan, typical flapping frequencies in the range of $10-15 \mathrm{~Hz}$ and a flight envelope ranging from near hover to fast forward flight. The mylar foil wings have an ' $\mathrm{X}$ '-configuration, allowing for increased lift production thanks to the clap-and-fling effect, while the T-shaped styrofoam tail leads to static stability and separates the bulk of the maneuvering from the wing flapping, operating according to the same principles as a standard aircraft tail, with movable rudder and elevator surfaces. The same type of vehicle has been the subject of extensive previous research and detailed geometric properties and descriptions of the design can be found, e.g., in Refs. [32, 31]. The mass and inertia properties of the specific FWMAV used in these flight tests are presented in Table 1. The inertia values were estimated from the mass and positioning of the separate components of the vehicle. Figure $2 \mathrm{~b}$ shows the orientation of the body-fixed coordinate system used in this study, defined to be centred at the center of gravity (CG) of the platform. In contrast to convention, the $z$-axis is aligned with the fuselage - this was done to avoid attitude singularities, given the typically large pitch angle assumed in flight by this FWMAV.

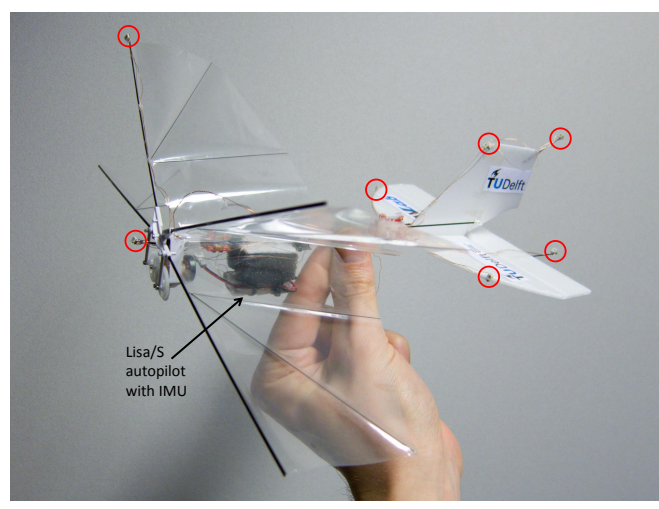

a) Test platform (DelFly II [32]), with LED markers for optical tracking circled.

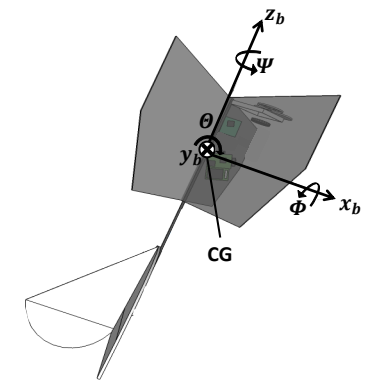

b) Body-fixed coordinate frame, centered at the CG; a typical flight attitude is shown (side view).

Figure 2: Test platform used in this study and body-fixed coordinate system $x_{b}, y_{b}, z_{b}$

\section{B. In-flight data acquisition}

The test platform was flown in a $10 \mathrm{~m} \times 10 \mathrm{~m} \times 7 \mathrm{~m}$ motion tracking facility (TU Delft Cyberzoo), equipped with 24 OptiTrack Flex13 cameras, and relevant measurements were acquired during flight using two different methods.

Firstly, the aforementioned optical tracking system (hereafter: "OptiTrack") was used to measure the positions of 7 active LED markers attached to the FWMAV at the locations illustrated in fig. 2a. The four markers attached to the fuselage and tail allowed for reconstruction of the position 
Table 1: Mass and inertia properties of the test platform

\begin{tabular}{ll}
\hline Property & Value \\
\hline$m[k g]$ & 0.0235 \\
$x_{C G}[m]$ (below fuselage) & 0.0096 \\
$y_{C G}[m]$ & 0 \\
$z_{C G}[m]$ (from wing leading edge) & -0.0695 \\
$I_{x x}, I_{y y}, I_{z z}\left[N m^{2}\right]$ & $7.5 \mathrm{E}-05,6.6 \mathrm{E}-05,1.9 \mathrm{E}-05$ \\
$I_{x y}, I_{y z}, I_{x z}\left[N m^{2}\right]$ & $0,0,8.5 \mathrm{E}-06$ \\
\hline
\end{tabular}

and orientation (quaternions) of the rigid body, while the remaining markers, on the wing leading edge, rudder and elevator allowed for reconstruction of the control surface deflections, and of the wing leading edge movement, hence the wing flap angle $(\zeta)$. The obtained measurements were transformed from the OptiTrack (i.e. inertial) coordinate system to the body-fixed coordinate frame shown in fig. 2b, and quaternions were transformed to Euler angles for easier interpretation. Note that the CG location does not coincide with the center of the OptiTrack-defined body-coordinate system, which is based on geometry. This difference was considered when processing the data (cf. also fig. 1). The LED markers have a mass of $0.035 \mathrm{~g}$ each, and high-speed imagery suggests that such a small mass has a negligible effect on the wing kinematics.

Secondly, on-board measurements were acquired by means of an inertial measurement unit (IMU). For the flight tests presented, the FWMAV was equipped with a $2.8 \mathrm{~g}$ Lisa/S autopilot [33], which includes a 6-axis MEMS IMU and a microcontroller unit (ARM Cortex-M3), allowing for open-source drone autopilot framework Paparazzi-UAV [34] to be run. The IMU was used to measure the linear accelerations and angular velocities of the vehicle, at a chosen sampling frequency of $512 \mathrm{~Hz}$. While sampling up to $1024 \mathrm{~Hz}$ is possible, internal low-pass filtering at $256 \mathrm{~Hz}$ implies that such sampling frequencies would not yield accurate additional information, and it was also found that at higher rates, significant numbers of frames were being lost, presumably as a result of CPU overloading. Moreover, previous work with similar ornithopters showed that very high-frequency content becomes increasingly difficult to distinguish from noise [16].

The IMU was attached to the body. Following the initial finding that the attachment mode influenced the measurements made, a block of foam was added between IMU and fuselage, to introduce damping and reduce unwanted vibrations. Small residual effects that remain despite the foam are being studied further, however these effects were found to be small after the aforementioned modification. To account for the fact that the IMU was not completely aligned with the body frame, a static calibration procedure was conducted prior to each flight. By comparing the accelerometer and optical tracking output at a number of different body orientations, the rotation between IMU and body frames was determined, and subsequently used to transform the in-flight IMU data to the body frame. 
The IMU measurements, as well as servo commands and motor speed, were logged using a microSD card added to the autopilot. Table 2 provides an overview of the measurements obtained, and Table 3 presents the main specifications of the IMU. Further details on the hardware and flight test setup can be found in [35].

Table 2: Overview of relevant data obtained from each data acquisition system.

\begin{tabular}{ll}
\hline Data acquisition means & Measurements provided \\
\hline \multirow{3}{*}{ OptiTrack } & Position $(x, y, z)$ \\
& Attitude quaternions $\left(q_{0}, q_{1}, q_{2}, q_{3}\right)$ \\
& Control surface deflections $\left(\delta_{E}, \delta_{R}\right)$ \\
& Wing flap angle $(\zeta)$ \\
\hline \multirow{2}{*}{ IMU } & Angular velocities $(p, q, r)$ \\
\hline \multirow{2}{*}{ On-board additional } & Linear accelerations $\left(a_{x}, a_{y}, a_{z}\right)$ \\
\hline & Servo commands $\left(\delta_{\mathrm{E}, \mathrm{CMD}}, \delta_{\mathrm{R}, \mathrm{CMD}}\right)$ \\
& Motor speed $(\mathrm{RPM})$ \\
\hline
\end{tabular}

Table 3: IMU specifications.

\begin{tabular}{lll}
\hline Parameter & Gyroscope & Accelerometer \\
\hline Measurement range & $\pm 2000^{\circ} / \mathrm{s}$ & $\pm 16 \mathrm{~g}$ \\
Sensitivity & $2 \%$ & $2 \%$ \\
Bandwidth* & $256 \mathrm{~Hz}$ & $256 \mathrm{~Hz}$ \\
Noise density & $0.005 \mathrm{deg} / \sqrt{\mathrm{Hz}}$ & $400 \mu \mathrm{g} / \sqrt{\mathrm{Hz}}$ \\
\hline
\end{tabular}

${ }^{*}$ As determined by the internal low-pass filtering, cf. sec.II. B

\section{Synchronization}

In order to be used together, the datasets given by IMU and OptiTrack, respectively, first had to be synchronized. This was done using one of the LED markers on the FWMAV body. This marker is switched on when the on-board logging starts, and off when it ends, thus allowing for synchronization of the measurement devices. However, the different sampling rates of the two data acquisition systems (120Hz for OptiTrack, $512 \mathrm{~Hz}$ for IMU), still entail a seemingly random time shift of up to $1 / 120 \mathrm{~s}=8.3 \mathrm{~ms}$ ( $10 \%$ of a flap cycle). To counter this effect, the time shift is estimated by shifting the IMU roll angle estimate by up to half a flap cycle (app. 41ms) in both directions until a minimum sum of squared errors is found between IMU and adequately resampled (using spline interpolation) OptiTrack signals. The data are then shifted accordingly prior to subsequent computations and filtering. The roll angle was used for synchronization because the best match between OptiTrack and IMU amplitude was observed in this angle, suggesting the roll axis is the most reliable, possibly due to the foam between IMU and body being stiffer in this direction. Additionally, in the current data there is limited excitation of the roll dynamics, and hence there 
are also less external factors influencing the time history of this variable. An alternative approach could be direct application of a multi-rate filter, accounting for the different sampling rates. This will be considered in future work.

\section{Data fusion}

\section{A. Motivation}

The flight test setup described allowed us to combine on-board IMU measurements and external optical tracking measurements, thus obtaining a highly accurate and informative dataset, suitable specifically for analyzing and modelling flapping-wing vehicles. In particular, this not only leads to the usual favorable results of sensor fusion, e.g. higher accuracy and reliability, but also allows for accurate high-frequency measurements, indispensable for in-depth analysis of flapping-wing flight and the short time-scale mechanisms involved. Additionally, unknown IMU biases can be estimated, further increasing the overall accuracy.

Optical tracking yields precise (typical mean tracking error approximately $1-2 \mathrm{~mm}$ ) and reliable measurements, whose accuracy does not deteriorate over time. The main drawbacks include: (i) relatively low sampling frequencies for the considered purpose, e.g. here $120 \mathrm{~Hz}$; (ii) presence of glitches and occasionally untracked or misattributed markers, e.g. due to light disturbance; and (iii) exclusive measurement of position and attitude, meaning rates must be computed via numerical differentiation, which amplifies the noise by a factor of $\frac{1}{(\Delta t)^{n}}$, with $n$ the order of differentiation and $\Delta t$ the time step [16]. The low acquisition frequency, in particular, means that even for the relatively low flapping frequency of the studied ornithopter $(10-15 \mathrm{~Hz})$, only approximately $8-12$ measurements are made during each flap cycle. As can be seen in fig. 4, this leads to a relatively low resolution, hence accurate analysis at a sub flap-cycle time scale is possible only with great limitations.

By contrast, IMU measurements are typically gathered at very high sampling frequencies, here $512 \mathrm{~Hz}$. This represents a significant advantage for looking at the sub flap-cycle level. Additionally, IMU devices provide relatively accurate measurements over short time scales - all of which can be exploited to generally enhance the reliability of the resulting dataset. The major drawback is drift integration, as well as increased noise and the possibility of biases, which must be computed during calibration procedures and/or estimated in flight. Additionally, in the current experimental setup the way the IMU is attached to the body also represents a potential source of error. While it is possible to investigate the effects of the foam attachment, the extremely low mass of the platform, as well as its shape, implies that however the IMU is attached, there will be an effect that may influence the results. In this sense, an additional advantage of a fusion approach is that the impact of such effects can be minimized through comparison with optical tracking data.

While each of the used measurement devices has limitations, combining their output allows for improved reliability, thanks to redundant information, the availability of more direct measure- 
ments, fewer issues with noise propagation, glitches and missing data, and more accurate and informative high-frequency data. In the rest of this section the data fusion approach is outlined and the resulting data are briefly discussed.

\section{B. Filter design}

An extended Kalman filter (EKF) was designed to fuse the IMU and optical tracking-provided data. The EKF is an extension of the linear Kalman filter that is applicable to nonlinear systems, with the drawback that a global optimal solution cannot be guaranteed, and divergence may occur, e.g. due to inadequate initialization. Improvements can be attained e.g. by using iterated Kalman filters, however, for the present application, an EKF was found to provide accurate results. The EKF is documented comprehensively in the literature (e.g. [36]), thus only the main equations are given here, for a nonlinear state-space system of the form:

$$
\begin{aligned}
x\left(t_{k+1}\right) & =f\left(x_{k}, u_{k}, t_{k}\right)+g\left(u_{k}, t_{k}\right)+w\left(t_{k}\right) \\
z\left(t_{k}\right) & =h\left(x, u, t_{k}\right)+v\left(t_{k}\right)
\end{aligned}
$$

where $x$ denotes the states, $u$ the input, $z$ the measured output, $w$ the process noise and $v$ the measurement noise. The process and measurement noise are assumed to be white and Gaussian, and are characterized by covariance matrices $Q$ and $R$, respectively. The filter is then given by the following equations.

Prediction:

$$
\begin{gathered}
\hat{x}_{k+1, k}=\hat{x}_{k, k}+\int_{t_{k}}^{t_{k+1}} f\left(\hat{x}_{k, k}, u_{k}, t\right) d t \\
P_{k+1, k}=\Phi_{k+1, k} P_{k, k} \Phi_{k+1, k}^{T}+\Gamma_{k+1, k} Q_{k+1} \Gamma_{k+1, k}^{T}
\end{gathered}
$$

Innovation:

$$
\begin{gathered}
K_{k+1}=P_{k+1, k} H_{k}^{T}\left[H_{k} P_{k+1, k} H_{k}^{T}+R_{k+1}\right]^{-1} \\
\hat{x}_{k+1, k+1}=\hat{x}_{k+1, k}+K_{k+1}\left[z_{k+1}-h\left(\hat{x}_{k+1, k}, u_{k+1}\right)\right] \\
P_{k+1, k+1}=P_{k+1, k}-K_{k+1} H_{k} P_{k+1, k}^{T}
\end{gathered}
$$

where $\Phi$ and $\Gamma$ are the discretized state transition and input matrices for the linearized system at step $k, P$ is the estimated measurement error covariance matrix, $K$ is the Kalman gain, and $H$ is the Jacobian of the measurement equation.

The filter designed in this study estimates the body attitude and velocities, as well as accelerometer and gyro biases, using IMU data as input variables and OptiTrack data as output measurements. Given that on the test platform, as on most current FWMAVs, no on-board velocity measurement was available, the velocities fed to the EKF as measurements $\left(u_{b}^{*}, v_{b}^{*}, w_{b}^{*}\right)$ were in fact calculated from the OptiTrack-measured positions, via numerical differentiation and necessary coordinate 
transforms, using the attitude computed from the OptiTrack-measured quaternions. Assuming the OptiTrack reference frame also has an upwards-pointing $z$-axis, the body-frame velocities are given by:

$\left[\begin{array}{c}u_{b}^{*} \\ v_{b}^{*} \\ w_{b}^{*}\end{array}\right]=\left[\begin{array}{ccc}\cos \Psi \cos \Theta & \cos \Theta \sin \Psi & -\sin \Theta \\ \cos \Psi \sin \Phi \sin \Theta-\cos \Phi \sin \Psi & \cos \Phi \cos \Psi+\sin \Phi \sin \Psi \sin \Theta & \cos \Theta \sin \Phi \\ \sin \Phi \sin \Psi+\cos \Phi \cos \Psi \sin \Theta & \cos \Phi \sin \Psi \sin \Theta-\cos \Psi \sin \Phi & \cos \Phi \cos \Theta\end{array}\right]\left[\begin{array}{c}\dot{x} \\ \dot{y} \\ \dot{z}\end{array}\right]_{O T}$

The required numerical derivatives were computed using a three point central difference method. The Euler angles were calculated from the quaternions which the OptiTrack software computes from the marker positions. Nonetheless, the attitude data were considered measurements, as they are a direct output of the OptiTrack. Moreover, the conversion from quaternions to Euler angles can be considered a matter of notation. The states, inputs, outputs and noise terms of the filter, according to eqs. $3-4$, are thus,

$$
\begin{aligned}
& x=\left[\begin{array}{llllllllllll}
\Phi & \Theta & \Psi & u_{b} & v_{b} & w_{b} & b_{p} & b_{q} & b_{r} & b_{a x} & b_{a y} & b_{a z}
\end{array}\right]^{T} \\
& u=\left[\begin{array}{llllll}
p & q & r & a_{x} & a_{y} & a_{z}
\end{array}\right]^{T} \\
& z=\left[\begin{array}{llllll}
\Phi_{m} & \Theta_{m} & \Psi_{m} & u_{b}^{*} & v_{b}^{*} & w_{b}^{*}
\end{array}\right]^{T} \\
& v=\left[\begin{array}{llllll}
v_{\Phi} & v_{\Theta} & v_{\Psi} & v_{u b} & v_{v b} & v_{w b}
\end{array}\right]^{T} \\
& w=\left[\begin{array}{llllll}
w_{p} & w_{q} & w_{r} & w_{a x} & w_{a y} & w_{a z}
\end{array}\right]^{T}
\end{aligned}
$$

and the process and measurement equations are:

Process equations:

$$
\begin{aligned}
\dot{\Phi} & =\left(p-b_{p}\right)+\left(q-b_{q}\right) \sin \Phi \tan \Theta+\left(r-b_{r}\right) \cos \Phi \tan \Theta \\
\dot{\Theta} & =\left(q-b_{q}\right) \cos \Phi-\left(r-b_{r}\right) \sin \Phi \\
\dot{\Psi} & =\left(q-b_{q}\right) \sin \Phi \sec \Theta+\left(r-b_{r}\right) \cos \Phi \sec \Theta \\
\dot{u}_{B} & =\left(r-b_{r}\right) v_{b}-\left(q-b_{q}\right) w_{b}-g \sin \Theta+a_{x}-b_{a x} \\
\dot{v}_{B} & =-\left(r-b_{r}\right) u_{b}+\left(p-b_{p}\right) w_{b}+g \sin \Phi \cos \Theta+a_{y}-b_{a y} \\
\dot{w}_{B} & =\left(q-b_{q}\right) u_{b}-\left(p-b_{p}\right) v_{b}+g \cos \Phi \cos \Theta+a_{z}-b_{a z} \\
\dot{b}_{p} & =0 \\
\dot{b}_{q} & =0 \\
\dot{b}_{r} & =0 \\
\dot{b}_{a x} & =0 \\
\dot{b}_{a y} & =0 \\
\dot{b}_{a z} & =0
\end{aligned}
$$




\section{Measurement equations}

$$
\begin{aligned}
\Phi_{m} & =\Phi+v_{\Phi} \\
\Theta_{m} & =\Theta+v_{\Theta} \\
\Psi_{m} & =\Psi+v_{\Psi} \\
u_{b}^{*} & =u_{b}+v_{x} \\
v_{b}^{*} & =v_{b}+v_{y} \\
w_{b}^{*} & =w_{b}+v_{z}
\end{aligned}
$$

where $[\Phi, \Theta, \Psi]$ are the Euler angles, $[p, q, r]$ are the roll, pitch and yaw rates, $\left[u_{b}, v_{b}, w_{b}\right]$ are the body-frame velocities, $\left[a_{x}, a_{y}, a_{z}\right]$ are the accelerometer-measured accelerations, $\left[b_{p}, b_{q}, b_{r}, b_{a x}, b_{a y}, b_{a z}\right]$ are the sensor biases of the gyroscope and accelerometer, $\left[v_{u b}, v_{v b}, v_{w b}, v_{\Phi}, v_{\Theta}, v_{\Psi}\right]$ are the measurement noise in the measured velocities and attitudes, and $\left[w_{p}, w_{q}, w_{r}, w_{a x}, w_{a y}, w_{a z}\right]$ are the process noise in the rates and accelerations. Asterisk superscripts indicate terms that are not directly measured but obtained from OptiTrack measurements, as discussed.

The process and measurement noise matrices, Q and R, were initially based on the actual noise characteristics of the available measurements. In a subsequent step, the matrices were adjusted further to achieve more effective results, based on the known or expected behavior of OptiTrack and IMU (cf. sec. III.A), on the desired outcome (i.e. accurate results also at relatively high frequencies), and (iteratively) on intermediate results obtained. Based on the previous discussion, a desirable solution is one where sufficient high-frequency content is left in the fused data, thanks to the IMU contribution, while the slow time-scale evolution largely follows that of the OptiTrack. Thus the underlying idea is to use the optical tracking data as a basis, particularly on a cycleaveraged and long-term level, while using IMU data to (i) compensate for glitches in the tracking and (ii) provide accurate high-frequency content. Additionally, the IMU provides useful direct measurements of the rates and accelerations, unavailable from the tracking system.

The primary goal in further tuning the $\mathrm{Q}$ and $\mathrm{R}$ matrices was to ensure a sufficiently strong preference for the OptiTrack, except at high frequencies, while also retaining as realistic results as possible. Hence, adjustments were made in order to give additional weight to the OptiTrack data. In particular, the R matrix was chosen to be slightly smaller (finally: $\times 0.8$ ) than the noise characteristics of the OptiTrack, as estimated from measurements, suggested. On the other hand, the $\mathrm{Q}$ matrix entries were selected to be somewhat larger (finally: $\times 2$ ) than what inferred from the data, to reduce the confidence in the IMU.

Within the range of values where the resulting flap-averaged time evolution approximately corresponded to that of the OptiTrack, the final values, i.e. the extent to which the baseline matrices estimated from the data were adjusted, were selected experimentally, by considering, firstly, the amplitude of the resulting flapping-related oscillations, and, secondly, the innovation errors and 
convergence behavior. The first point involved ascertaining that the high-frequency content was still predominantly taken over from the IMU. Prior to this, preliminary evaluations were made to investigate to what extent the IMU high-frequency data are reliable. It was observed that in some cases the flapping-related oscillations had larger amplitudes in IMU than in OptiTrack measurements. This may be due to the effect of the IMU mount, and, for the velocities, different sensitivities of the accelerometer along different axes may also have an effect. Nonetheless, comparing forces estimated from the IMU alone to wind tunnel data [35] showed an overall adequate agreement, with minor discrepancies. While no true values are available for validation, and there are known to be some differences between wind tunnel and free-flight forces [13], this observation nonetheless suggests that at sub-flap cycle level, the IMU information can be considered sufficiently reliable.

The second step involved comparing the innovation errors to the uncertainty bounds predicted by the EKF. It was found that while reducing $R$ and increasing $Q$ leads to increasingly small innovation errors and faster filter convergence, this can eventually lead to a significant discrepancy between innovation errors and error bounds predicted by the filter. Thus, a compromise was found, where the errors and estimated error bounds are small, but the former are still largely contained within the latter. Even a very significant higher weighting of the OptiTrack measurements (cf. eqs. 26, 27) was found to allow for high-frequency content to be taken over from the IMU measurements, while also leading to small innovation errors and rapid convergence.

The final covariance matrices were set to the following values.

$$
\begin{aligned}
& Q=\operatorname{diag}(3.06,7.30,7.12,44.21,14.80,24.20) \\
& R=\operatorname{diag}(9.94 E-4,1.07 E-3,8.33 E-4,9.80 E-3,2.47 E-3,6.82 E-3)
\end{aligned}
$$

\section{Results}

\section{A. Fusion results}

Figure 3 shows an example of fused data, compared to data obtained from the OptiTrack and from the IMU directly. Note that the IMU-calculated values are still computed using OptiTrack measurements for the initial conditions and to convert to the body frame where required. It can be seen that an accurate result is achieved. In the long term there is significant agreement between the OptiTrack data and the EKF result, avoiding typical IMU drawbacks such as drift integration that lead to inaccurate attitude and position reconstruction. At small time scales, on the other hand, the IMU sensor readings are used to obtain a high-resolution, accurate result, that improves significantly on what could be achieved with the the OptiTrack alone. The detailed views in fig. 4 further highlight the increased information that can be obtained at high frequencies. Note that in 
the closest view provided in subfig. 4a the gyro measurement is no longer visible as it has drifted above the other two signals, as seen in the remaining subfigures. The overall result can also be evaluated in the frequency domain, e.g. using fast Fourier transforms (FFT), as for instance shown in fig. 5 for the pitch attitude. Here it can be clearly seen which measurement source is favored in which frequency range.

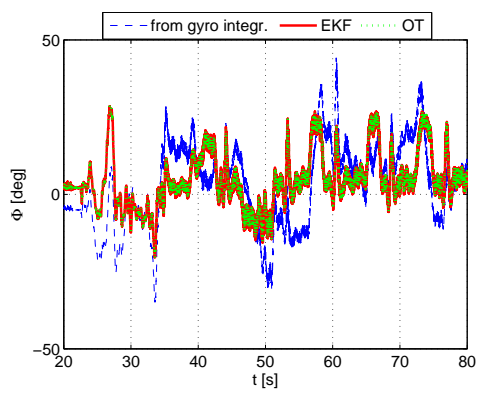

a) $\Phi$

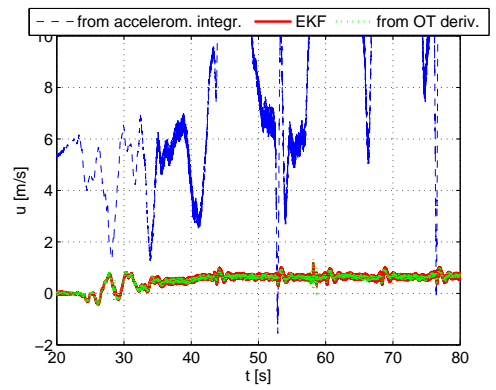

d) $u_{b}$

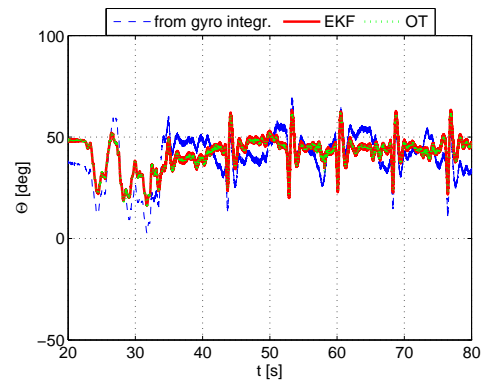

b) $\Theta$

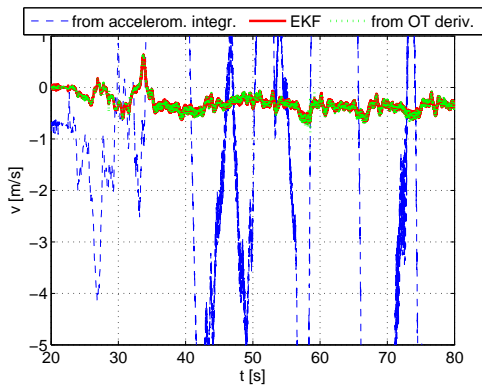

e) $v_{b}$

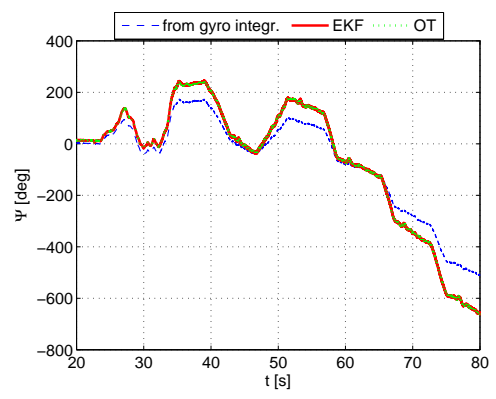

c) $\Psi$

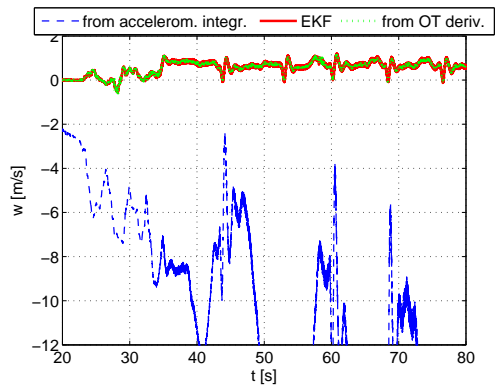

f) $w_{b}$

Figure 3: Comparison of OptiTrack (OT) measurements, IMU integration-obtained values, and EKFobtained values, during free flight.

Figure 6 shows that the innovation errors $\left(z_{k+1}-h\left(\hat{x}_{k+1, k}, u_{k+1}\right)\right.$, cf. eq. 6$)$ are mostly contained within the estimated error bounds, suggesting an effective filter implementation. As can be observed in the figure, the errors in the estimated attitude are typically approximately $1-2^{\circ}$ during flight, and below $0.2^{\circ}$ in the static case, however errors in the velocities are still relatively high during flight, reaching up to $0.1 \mathrm{~m} / \mathrm{s}$, possibly due to the numerical derivatives used as measurements in eqs. 23-25. As can be observed in the plots, errors are also slightly higher during dynamic maneuvers, however they either remain within the estimated bounds, or venture only slightly beyond these. More effective tuning will be explored to improve this result further.

Bias terms (cf. eqs. 8-19) were found to converge in the static case, while during flight they vary to a limited extent, typically in conjunction with maneuvers. It can for instance be observed in fig. 8, that the bias estimates, particularly the ones for the lateral acceleration measurement, change somewhat when turns are being flown (i.e. in conjunction with long rudder input signals). To some extent, these changes may reflect a real, small movement of the IMU relative to the body, which cannot be excluded in the current setup. Nonetheless, these effects are at a very small 

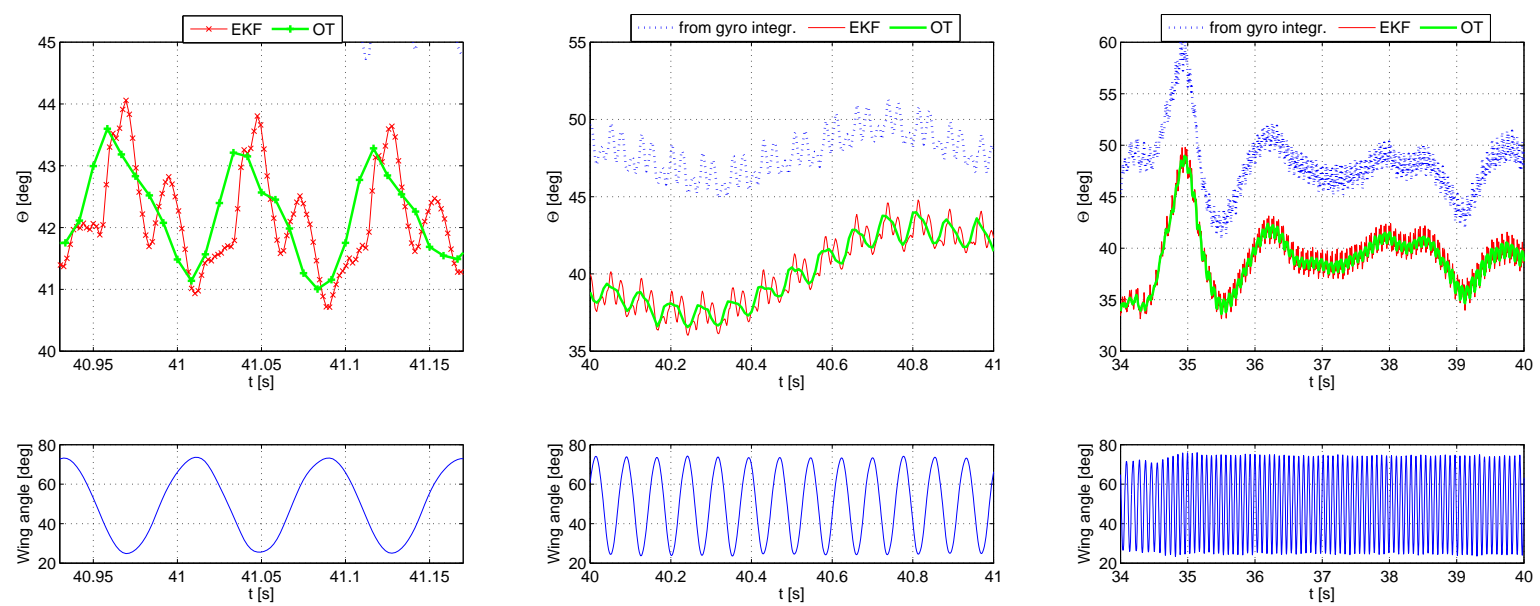

a) $t \in[40.93,41.17] \mathrm{s}$

b) $t \in[40,41] \mathrm{s}$

c) $t \in[34,40] s$

Figure 4: Detail of EKF and OptiTrack-obtained results: high-frequency content in pitch attitude, with corresponding wing flap angle below (increasingly small time scale view of the same measurements from right to left)

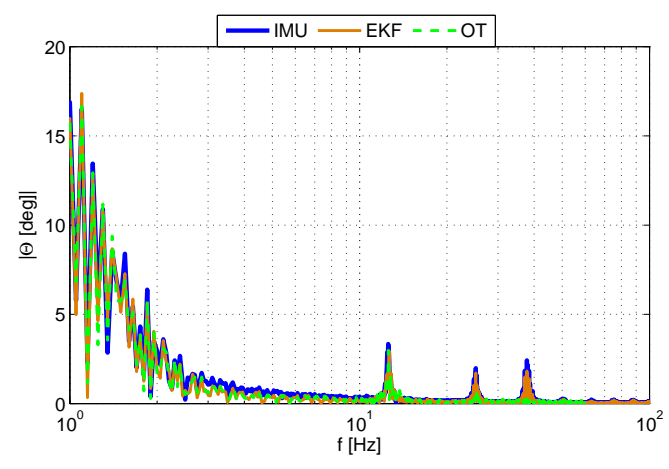

a) Full view

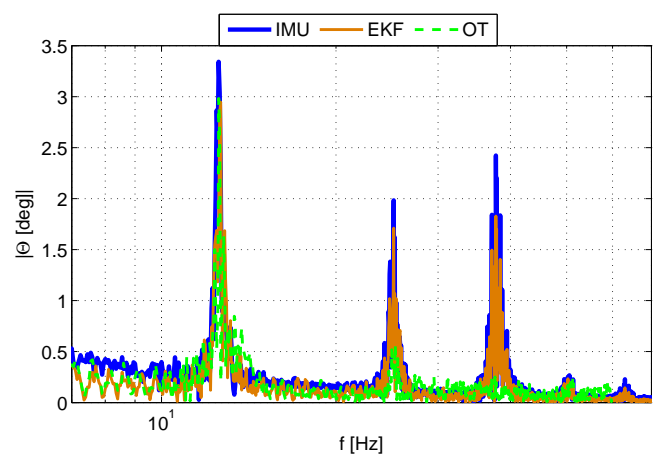

b) Detailed view

Figure 5: FFT of the pitch attitude computed from OptiTrack, EKF and IMU

scale, and typically only connected to large, long-lasting maneuvers, whereas rapid longitudinal inputs mostly appear to have only a slight transient effect. The similar bias values estimated from different datasets (cf. fig. 7), suggest reliable results, and all the values were found to be small, so that neglecting the bias terms may not have a significant impact. Nonetheless, the possibility of biases should be considered in a general setup. Indeed, the possibility of estimating such biases is another advantage of using data fusion.

Further testing and analysis of the sensors is required to draw conclusions on the remaining discrepancies between IMU and OptiTrack measurements mentioned above. Additionally, the velocity estimation can still be improved, e.g. by further fine-tuning of the filter and perhaps the use of higher-order differencing schemes and perhaps the use of higher-order differencing schemes to compute the velocities from the position data. The current setup should however be suitable for 

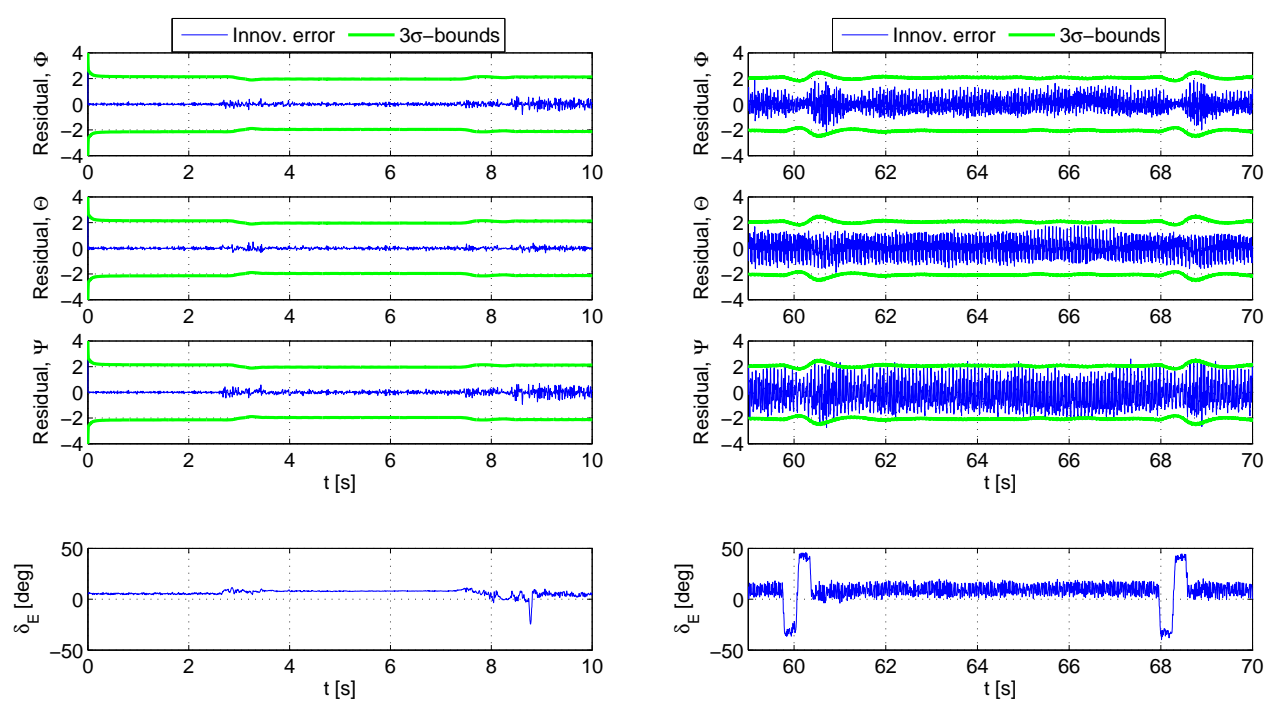

a) Attitude, static condition

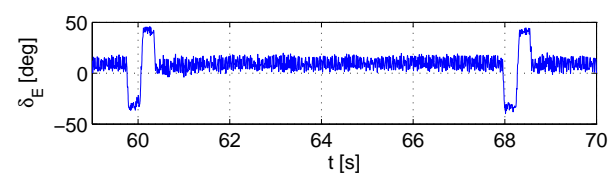

b) Attitude, free flight
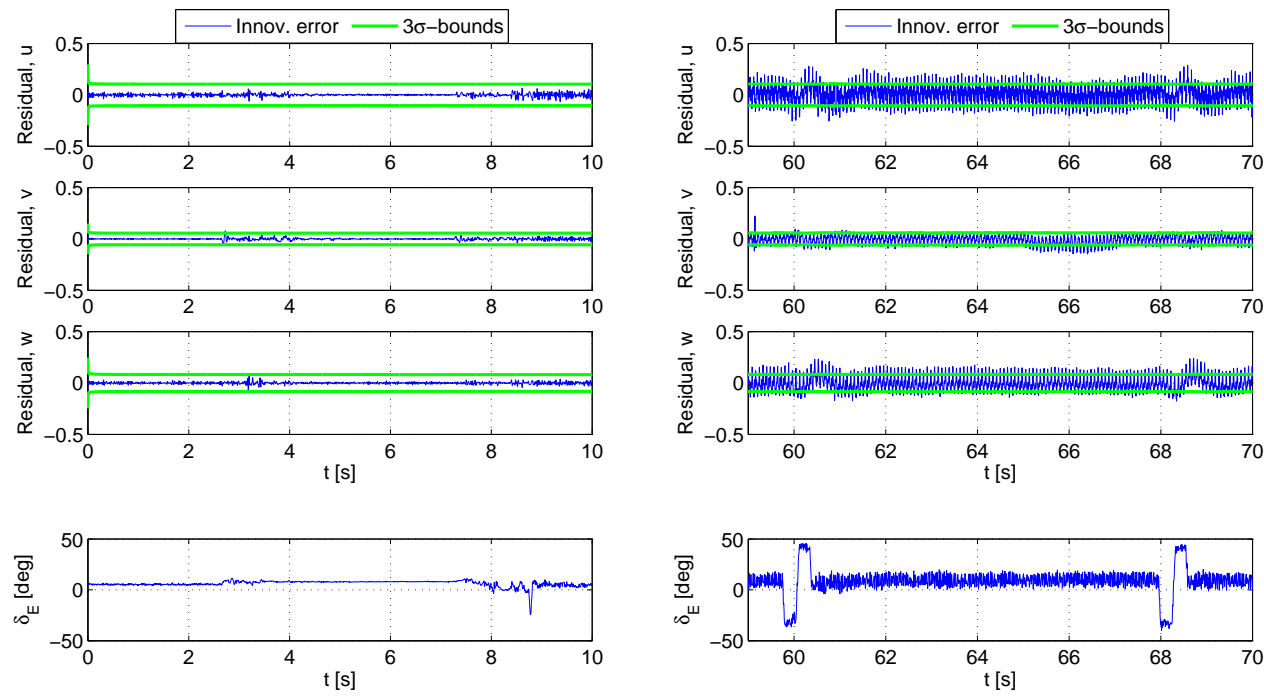

c) Velocity, static condition

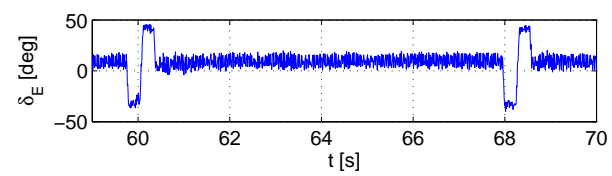

d) Velocity, free flight

Figure 6: EKF innovation errors and predicted error variance bounds $( \pm 3 \sigma)$ in static and free flight segments; and corresponding measured elevator deflection.

most modelling and identification work. The obtained data are accurate, contain detailed highfrequency information, and entail increased reliability due to the merging of separate data sources.

\section{B. Outlook for time-resolved flapping flight analysis}

The suggested experimental setup and data fusion approach allow for effective analysis of sub-flap cycle processes. It becomes possible, in free flight, to consider higher frequency content than can be achieved with optical tracking alone (cf. fig. 4), and to consider dynamic maneuvers, which is not possible in open-loop wind tunnel experiments.

Accurate and realistic high-frequency content can be useful for dynamic analysis, e.g. consid- 


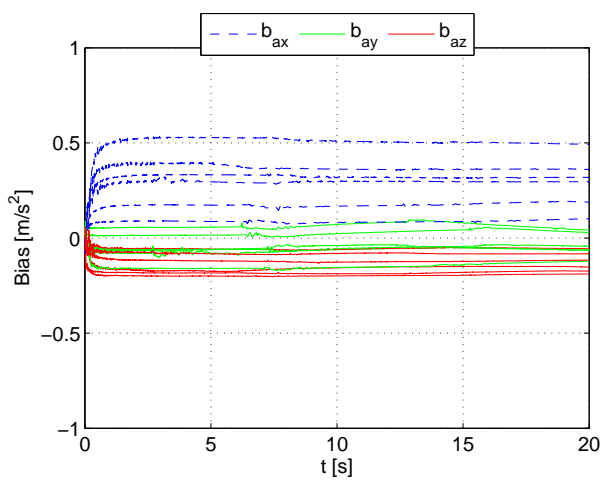

a) Accelerometer bias, static

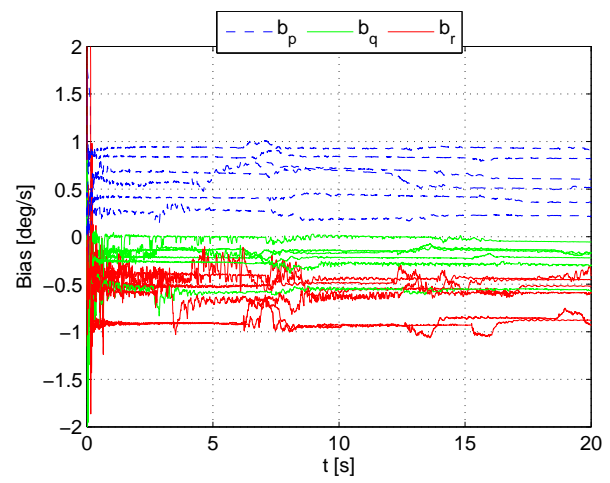

c) Gyroscope bias, static

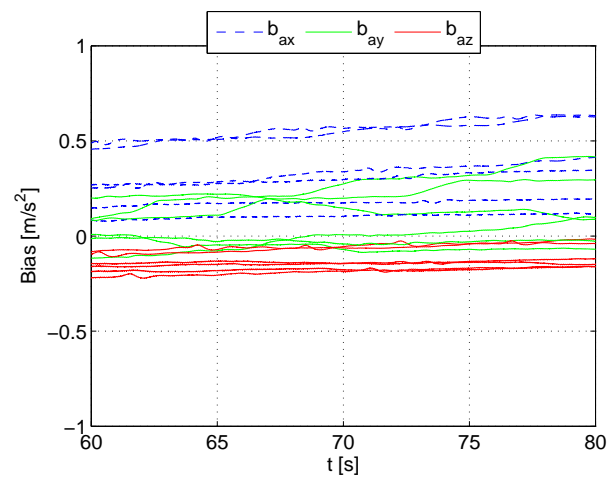

b) Accelerometer bias, free-flight

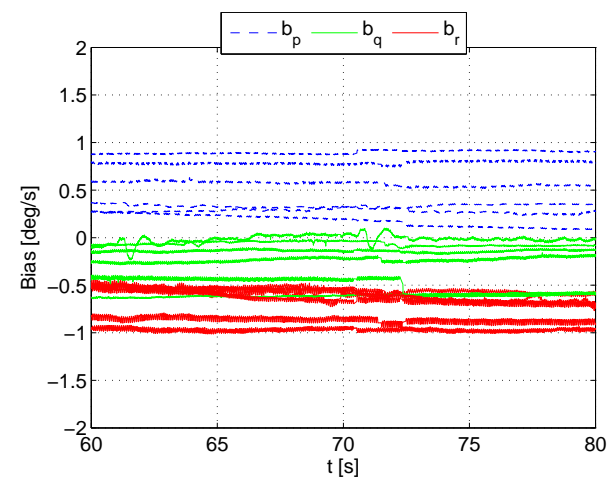

d) Gyroscope bias, free-flight

Figure 7: Estimated sensor biases, in static conditions and during manoeuvring flight (for six datasets)
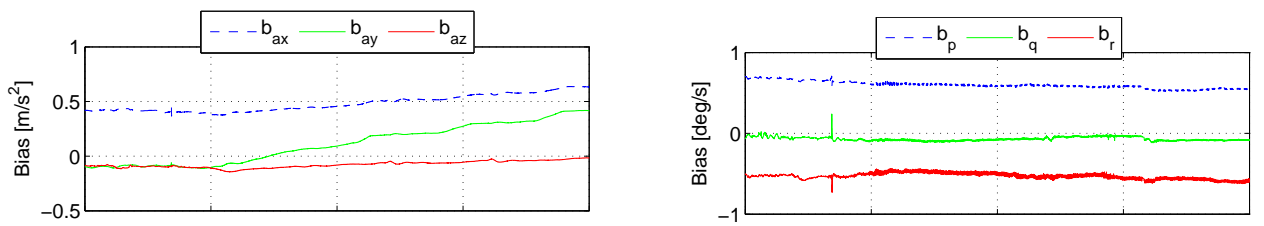

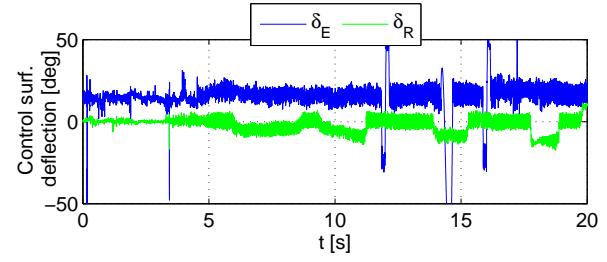

a) Accelerometer bias

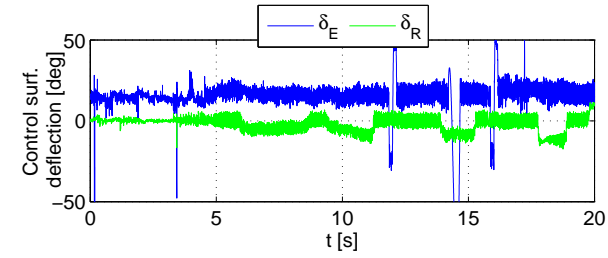

b) Gyroscope bias

Figure 8: Estimated sensor biases for a single dataset (free-flight conditions) and corresponding measured elevator deflection.

ering the effect of the flapping-related oscillations in the states, however it is particularly essential in aerodynamic studies. It has for instance been shown that some unsteady effects, such as clapand-fling, are visible clearly only at very high frequencies [14], which are typically only accurately observable in wind tunnel tests. At the same time, as mentioned in sec. I, wind tunnel and freeflight data are only identical in limited and very specific cases[16], hence it is valuable to have the 
ability to obtain qualitatively comparable data in free-flight conditions. The suggested filter yields results that are close to wind tunnel results in terms of detail and information content, while also more realistic and practically useful, as they represent free flight.

As an example, figs. 9 and 10 show a comparison of the aerodynamic forces in body frame $x$ and $z$ axes (i.e. the forces relevant for longitudinal maneuvers) computed from EKF data, OptiTrack data and wind tunnel data, for four different flight conditions. The wind tunnel measurements [35] were conducted in conditions similar to the conditions observed in flight (in terms of angle of attack, forward velocity and flapping frequency), however it must be considered that small differences in the test conditions are inevitable, given the experimental setting. The data are shown for two different filter cutoff frequencies, chosen just above three times and five times the flapping frequency, respectively. In the considered range of flight conditions, flapping frequencies vary between approximately $10 \mathrm{~Hz}$ and $14 \mathrm{~Hz}$. The plots show two flap cycles, and the data are plotted against nondimensional time, $t^{*}:=t / T_{\text {cycle }}$. Qualitatively, it can be seen that there is consider-
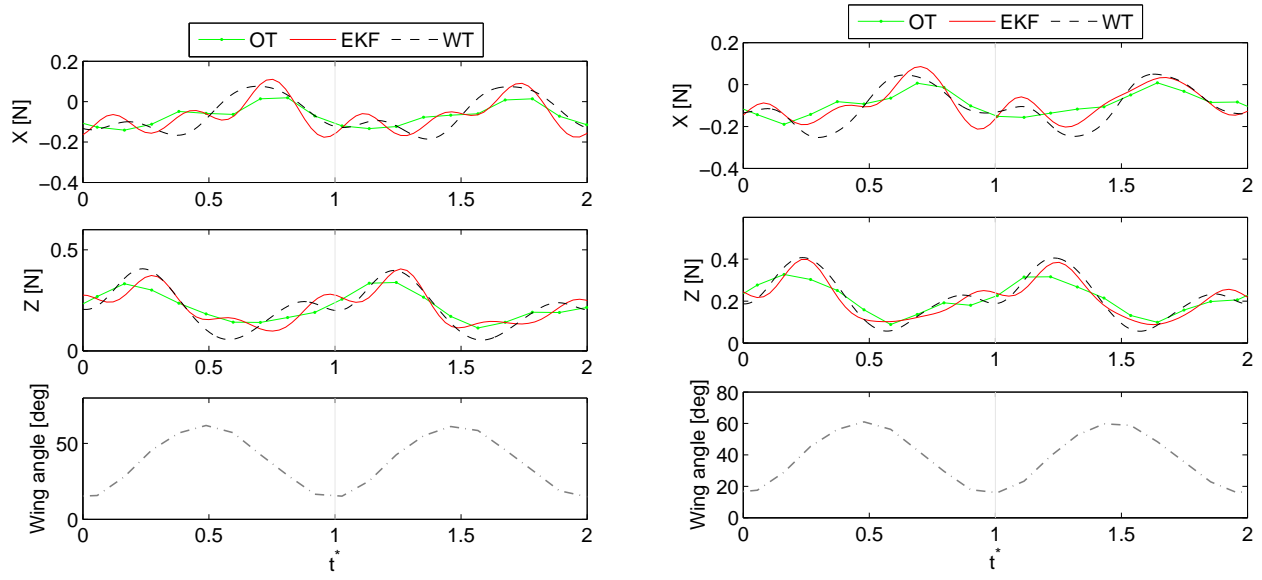

a) $V \approx 0.4 \mathrm{~m} / \mathrm{s}$

b) $V \approx 0.7 \mathrm{~m} / \mathrm{s}$
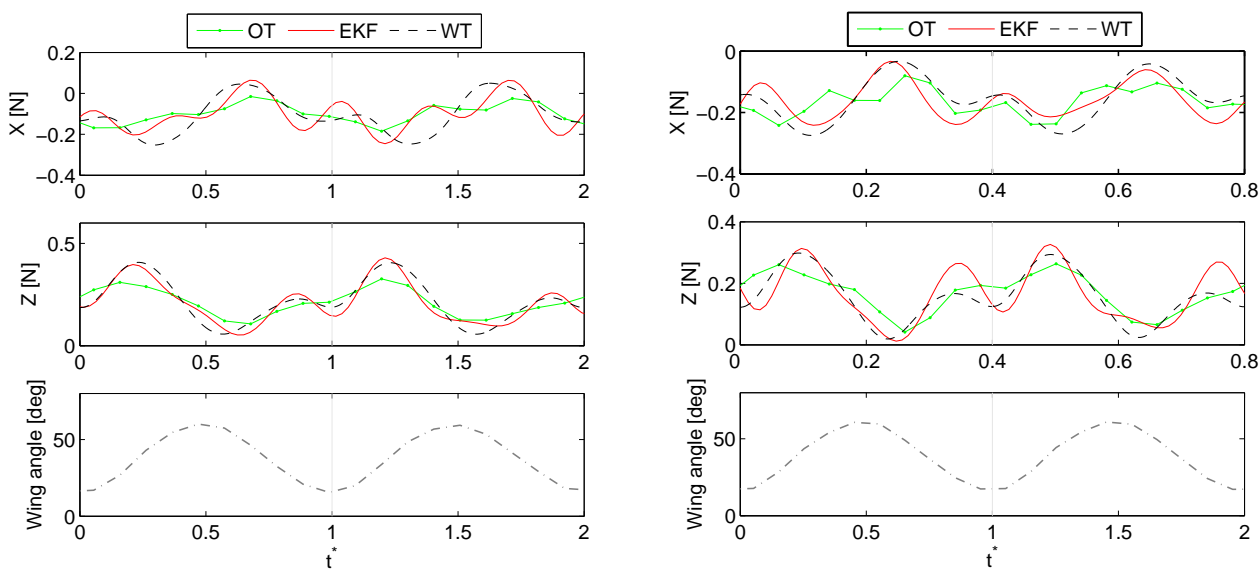

c) $V \approx 0.8 \mathrm{~m} / \mathrm{s}$

d) $V \approx 1.3 \mathrm{~m} / \mathrm{s}$

Figure 9: Aerodynamic forces obtained from OptiTrack (OT), EKF and wind tunnel (WT); low-pass filtered above the third flapping frequency harmonic. 

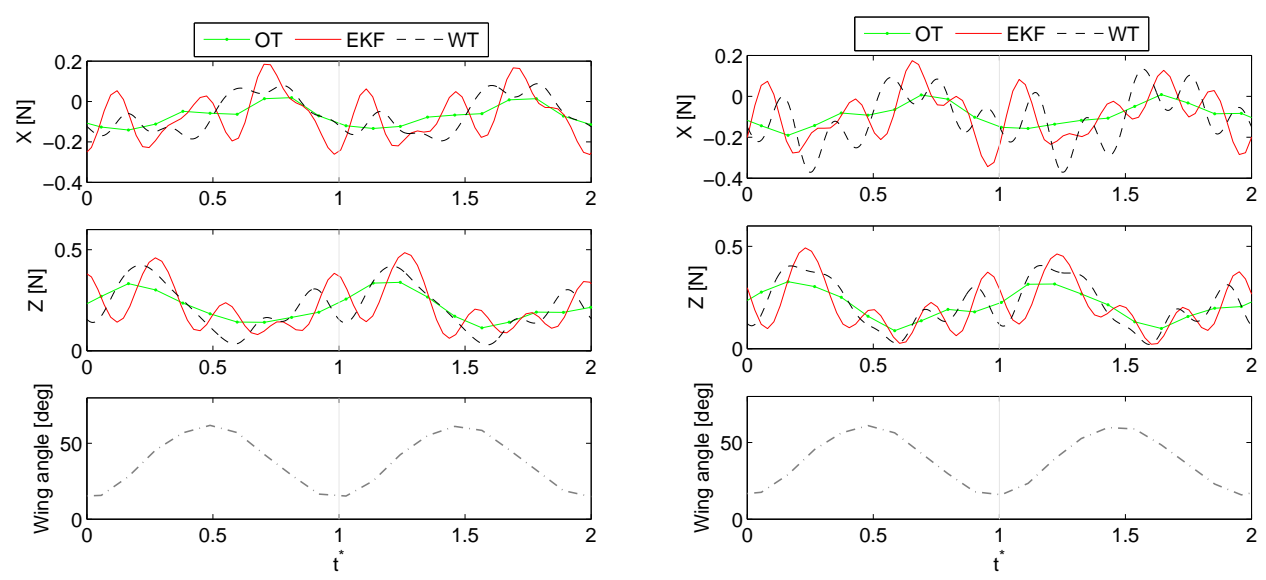

a) $V \approx 0.4 m / s$

b) $V \approx 0.7 \mathrm{~m} / \mathrm{s}$
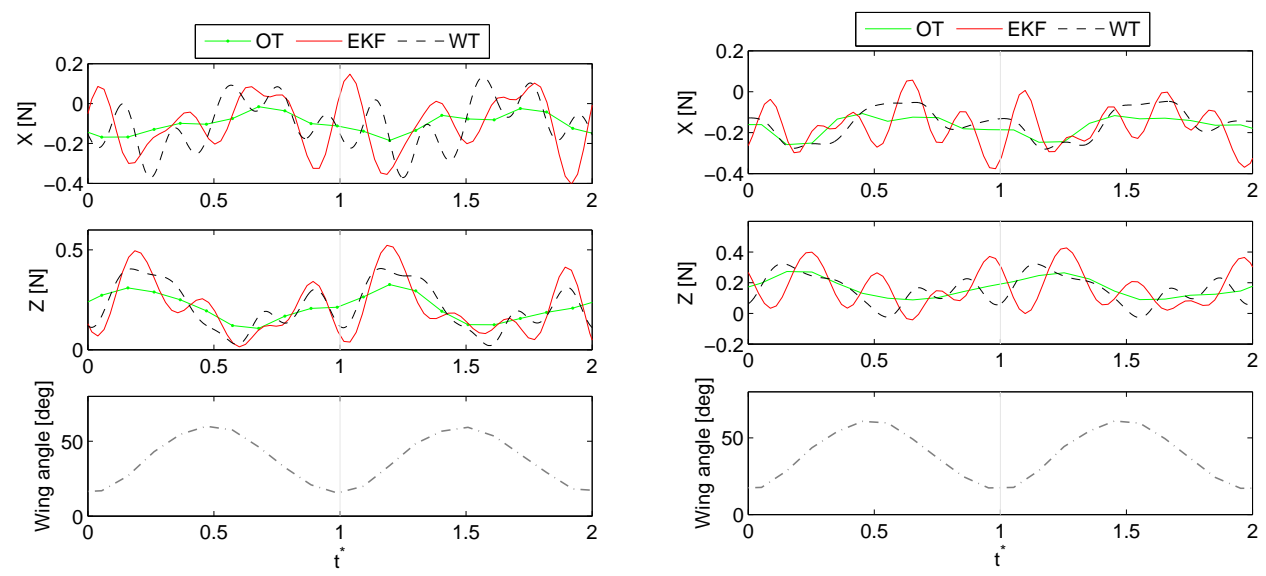

c) $V \approx 0.8 \mathrm{~m} / \mathrm{s}$

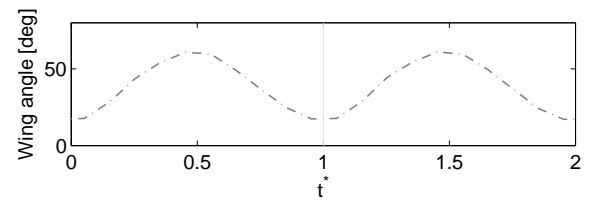

d) $V \approx 1.3 \mathrm{~m} / \mathrm{s}$

Figure 10: Aerodynamic forces obtained from OptiTrack (OT), EKF and wind tunnel (WT); low-pass filtered above the fifth flapping frequency harmonic.

able agreement between EKF and wind tunnel forces, particularly for the force component parallel to the fuselage $(Z)$. Slight discrepancies, particularly in the normal force component $(X)$, are in line with previous results suggesting differences between wind tunnel and free-flight forces in this component particularly [16]. In fact the wind tunnel and free-flight cases cannot be considered entirely equal, as the clamping has a significant impact on the recorded forces [16]. Hence complete equality cannot be expected even in an ideal case. Nonetheless, the considerable agreement suggests that the remaining discrepancies between wind tunnel and fused free flight forces are at least partly due to actual differences between the free-flight and the clamped wind tunnel situation, rather than due to measurement error. This is also suggested by the similar trend in the forces measured for different flight conditions, in wind tunnel and free flight data. In terms of information content, the EKF and wind tunnel forces display a similar resolution and smoothness, and indeed can be considered of comparable quality. By contrast, the OptiTrack derived forces have a visibly lower resolution, as evident from the more abrupt and discontinuous evolution. While the evolu- 
tion tendentially echoes that of the EKF and wind tunnel, details are not recognizable due to the insufficient number of measurement points. Clearly, a higher sampling frequency is required for accurate capturing of sub-flap forces in free flight. It can be seen (cf. fig. 10) that even with relatively high filter cut-off frequencies, leaving five flapping frequency harmonics in the data, there is still a considerable level of agreement between wind tunnel and EKF results, and a comparable level of detail, suggesting that the suggested data fusion approach allows for high frequencies to be considered.

Finally, the possibility of considering maneuvers is also of interest, as maneuvering flight is essential for a real flying vehicle, and particularly for flapping-wing flyers, which boast high maneuverability as one of their pivotal advantages. Furthermore, previous work has hypothesized that additional interactions may occur at the sub-flap level during maneuvers [25, 37], that are not observable in steady flight. Again, this cannot be studied in the wind tunnel.

Thus, the suggested combination of different data sources yields a more comprehensive and in-depth overview of time-resolved flapping behavior that constitutes a useful stepping stone for further work in this field.

$\longrightarrow V=0.4 \mathrm{~m} / \mathrm{s}-\mathrm{V}=0.7 \mathrm{~m} / \mathrm{s}---V=0.9 \mathrm{~m} / \mathrm{s}-V=1.1 \mathrm{~m} / \mathrm{s} \cdots \cdots, V=1.3 \mathrm{~m} / \mathrm{s}$
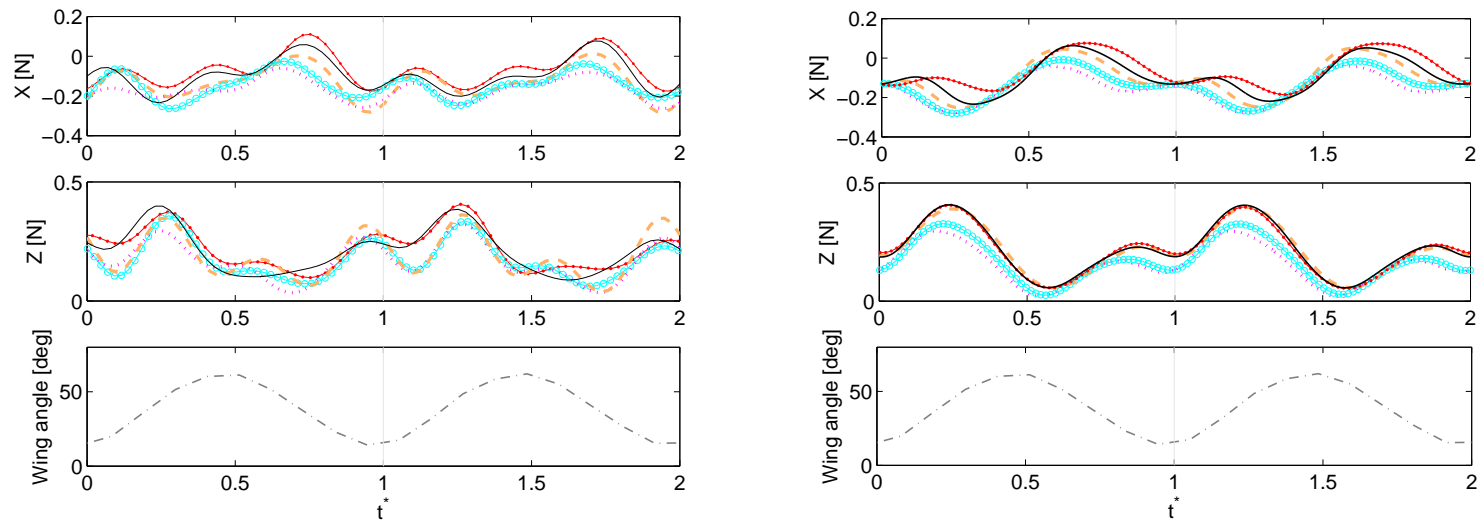

a) Forces from fused free-flight data

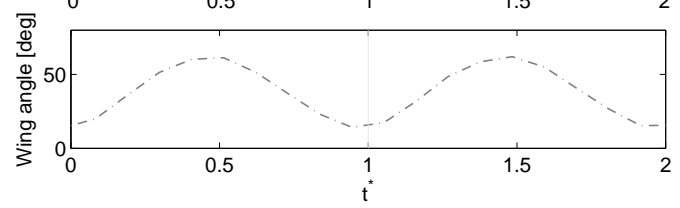

b) Forces from wind tunnel data

Figure 11: Aerodynamic forces for different total velocities; filtering above the third flapping frequency harmonic.

\section{Conclusion}

An extended Kalman filter was suggested to fuse data from on-board inertial sensors and offboard optical tracking measurements on a flapping-wing micro aerial vehicle (FWMAV). Compared to both the separate measurement methods, this approach was found to provide more reliable and accurate data and, in particular, to yield more extensive and accurate insight into the behavior of the vehicle during each flap cycle, in terms of both the vehicle's states and the forces acting on 
it. Other advantages include the possibility of estimating the velocities more effectively than with either the inertial measurement unit (IMU) or optical tracking alone, or the possibility of obtaining accurate attitude data also for very small platforms, where the information provided by tracking alone decreases in quality due to the limited resolution.

The proposed approach is considered generally useful for studying flapping-wing vehicles in free flight, as it avoids the tethering inherent in wind tunnel testing and also allows for maneuvering and different flight conditions to be easily considered, while still providing a comparable degree of detail. Both slow and fast time-scale processes can be captured in detail, supporting the modelling process and providing better insight into the properties of novel, flapping-wing vehicles. The method is expected to remain effective also for smaller vehicles. Finally, while the suggested data fusion setup can only be implemented offline, with the considered instrumentation, it allows for more accurate and detailed models to be constructed and validated, and therefore can be considered a useful basis also for advanced, sub-flap cycle control in the future.

\section{References}

[1] Ellington, C. P., "The Aerodynamics of Hovering Insect Flight. I. The Quasi-Steady Analysis," Philosophical Transactions of the Royal Society B: Biological Sciences, Vol. 305, No. 1122, Feb. 1984, pp. 1-15. doi:10.1098/rstb.1984.0049.

[2] Ellington, C., van den Berg, C., Willmott, A., and Thomas, A., "Leading-edge vortices in insect flight," Nature, Vol. 384, No. 19/26, 1996, pp. 626-630. doi:10.1038/384626a0.

[3] Taylor, G. K., "Dynamic flight stability in the desert locust Schistocerca gregaria," Journal of Experimental Biology, Vol. 206, No. 16, Aug. 2003, pp. 2803-2829. doi:10.1242/jeb.00501.

[4] Sane, S. P., "The aerodynamics of insect flight," Journal of Experimental Biology, Vol. 206, No. 23, Dec. 2003, pp. 4191-4208. doi:10.1242/jeb.00663.

[5] Dickinson, M. H., Lehmann, F., and Sane, S. P., "Wing Rotation and the Aerodynamic Basis of Insect Flight," Science, Vol. 284, No. 5422, June 1999, pp. 1954-1960. doi:10.1126/science.284.5422.1954.

[6] Dietl, J., Herrmann, T., Reich, G., and Garcia, E., "Dynamic Modeling, Testing, and Stability Analysis of an Ornithoptic Blimp," Journal of Bionic Engineering, Vol. 8, No. 4, Dec. 2011, pp. 375-386. doi:10.1016/S1672-6529(11)60043-7.

[7] Doman, D. B., Oppenheimer, M. W., and Sigthorsson, D. O., "Dynamics and Control of a Minimally Actuated Biomimetic Vehicle: Part I - Aerodynamic Model," AIAA Guidance, Navigation, and Control Conference, 2009, AIAA Paper 2009-6160. doi:10.2514/6.2009-6160.

[8] Grauer, J., Ulrich, E., Jr., J. H., Pines, D., , and Humbert, J. S., "Testing and System Identification of an Ornithopter in Longitudinal Flight," Journal of Aircraft, Vol. 48, No. 2, March-April 2011, pp. 660667. doi:10.2514/1.C031208.

[9] Han, J.-S., Chang, J. W., Kim, J.-k., and Han, J.-H., "Experimental Study on the Unsteady Aerodynamics of a Robotic Hawkmoth Manduca sexta model," AIAA SciTech 13-17 January 2014, National Harbor, Maryland, 52nd Aerospace Sciences Meeting, 2014, AIAA Paper 2014-1113. doi:0.2514/6.20141113. 
[10] Lee, J.-S. and Han, J.-H., "Experimental study on the flight dynamics of a bioinspired ornithopter: free flight testing and wind tunnel testing," Journal of Smart Materials and Structures, Vol. 21, 2012. doi:10.1088/0964-1726/21/9/094023.

[11] Nakata, T., Liu, H., Tanaka, Y., Nishihashi, N., Wang, X., and Sato, A., "Aerodynamics of a bio-inspired flexible flapping-wing micro air vehicle," Bioinspiration \& Biomimetics, Vol. 6, 2011. doi:10.1088/1748-3182/6/4/045002.

[12] Percin, M., Eisma, H., van Oudheusden, B., Remes, B., Ruijsink, R., and de Wagter, C., "Flow Visualization in the Wake of the Flapping-Wing MAV 'DelFly II' in Forward Flight,' AIAA Applied Aerodynamics Conference, 2012, AIAA Paper 2012-2968. doi:10.2514/6.2012-2664.

[13] Caetano, J. V., Weehuizen, M. B., de Visser, C. C., de Croon, G. C. H. E., and Mulder, M., "Rigid-Body Kinematics Versus Flapping Kinematics of a Flapping Wing Micro Air Vehicle," Journal of Guidance, Control and Dynamics, Vol. 38, No. 12, Dec. 2015, pp. 2257-2269. doi:10.2514/1.G000923.

[14] Armanini, S. F., Caetano, J., de Croon, G., de Visser, C., and Mulder, M., "Quasi-steady aerodynamic model of clap-and-fling flapping MAV and validation with free-flight data," Bioinspiration \& Biomimetics, Vol. 11, No. 4, 2016, pp. 046002. doi:10.1088/1748-3190/11/4/046002.

[15] DeLuca, A. M., Reeder, M. F., and Cobb, R. G., "An Experimental Investigation into the Effect of Flap Angles for a Piezo-Driven Wing," International Journal of Micro Air Vehicles, Vol. 5, No. 1, 2013. doi:10.1260/1756-8293.5.1.55.

[16] Caetano, J. V., Percin, M., van Oudheusden, B. W., Remes, B. D. W., de Wagter, C., de Croon, G. C. H. E., and de Visser, C. C., "Error Analysis and Assessment of Unsteady Forces Acting on a Flapping Wing Micro Air Vehicle: Free-Flight versus Wind Tunnel Experimental Methods," Bioinspiration \& Biomimetics, Vol. 10, No. 5, 2015. doi:10.1088/1748-3190/10/5/056004.

[17] Jennings, A., Mayhew, M., and Black, J., "Video measurements of instantaneous forces of flapping wing vehicles," Mechanical Systems and Signal Processing, Vol. 64-65, No. 2015, Dec. 2015, pp. 325336. doi:10.1016/j.ymssp.2015.04.020.

[18] Rose, C. and Fearing, R. S., "Comparison of Ornithopter Wind Tunnel Force Measurements with Free Flight," 2014 IEEE International Conference on Robotics \& Automation (ICRA), 2014, pp. 1816-1821. doi:10.1109/ICRA.2014.6907097.

[19] Grauer, J., Ulrich, E., Hubbard, J. E., Pines, D., and Humbert, J. S., “Testing and System Identification of an Ornithopter in Longitudinal Flight," Journal of Aircraft, Vol. 48, No. 2, March 2011, pp. 660667. doi:10.2514/1.C031208.

[20] Finio, B. M., Perez-Arancibia, N. O., and Wood, R. J., "System identification and linear time-invariant modeling of an insect-sized flapping-wing micro air vehicle," IEEE/RSJ International Conference on Intelligent Robots and Systems, 2011, pp. 1107-1114. doi:10.1109/IROS.2011.6094421.

[21] Duan, H. and Li, Q., "Dynamic model and attitude control of Flapping Wing Micro Aerial Vehicle," 2009 IEEE International Conference on Robotics and Biomimetics (ROBIO), Dec. 2009, pp. 451-456. doi:10.1109/ROBIO.2009.5420689.

[22] Chand, A. N., Kawanishi, M., and Narikiyo, T., "Parameter Estimation for the Pitching Dynamics of a Flapping-Wing Flying Robot," IEEE International Conference on Advanced Intelligent Mechatronics (AIM), Busan, Korea, 2015, pp. 1552-1558. doi:10.1109/AIM.2015.7222763. 
[23] Caetano, J. V., de Visser, C. C., de Croon, G. C. H. E., Remes, B. D. W., de Wagter, C., Verboom, J. L., and Mulder, M., "Linear Aerodynamic Model Identification of a FlappingWing MAV Based on Flight Test Data," International Journal of Micro Air Vehicles, Vol. 5, No. 4, December 2013, pp. 273-286. doi:10.1260/1756-8293.5.4.273.

[24] Armanini, S. F., de Visser, C. C., and de Croon, G. C. H. E., "Black-box LTI modelling of flappingwing micro aerial vehicle dynamics," AIAA Atmospheric Flight Mechanics Conference, Kissimee, FL, USA, 2015, AIAA Paper 15-0234. doi:10.2514/6.2015-0234.

[25] Armanini, S. F., de Visser, C. C., de Croon, G. C. H. E., and Mulder, M., "Time-varying model identification of flapping-wing vehicle dynamics using flight data," Journal of Guidance, Control, and Dynamics, Vol. 39, No. 3, 2016, pp. 526-541. doi:10.2514/1.G001470.

[26] Xiong, Y. and Sun, M., "Stabilization control of a bumblebee in hovering and forward flight," Acta Mechanica Sinica, Vol. 25, No. 1, 2009, pp. 13-21. doi:10.1007/s10409-008-0184-8.

[27] Wu, J. and Sun, M., "Control for going from hovering to small speed flight of a model insect," Acta Mechanica Sinica, Vol. 25, No. 3, 2009, pp. 295-302. doi:10.1007/s10409-009-0241-y.

[28] Berman, G. J. and Wang, Z. J., "Energy-minimizing kinematics in hovering insect flight," Journal of Fluid Mechanics, Vol. 582, June 2007, pp. 153-168. doi:10.1017/S0022112007006209.

[29] Orlowski, C. T. and Girard, A. R., "Averaging of the Nonlinear Dynamics of Flapping Wing Micro Air Vehicles for Symmetrical Flapping," AIAA Journal, Vol. 49, No. 5, May 2011, pp. 969-981. doi:10.2514/1.J050649.

[30] Chirarattananon, P. and Wood, R. J., "Identification of flight aerodynamics for flapping-wing microrobots," 2013 IEEE International Conference on Robotics and Automation, May 2013, pp. 1389-1396. doi:10.1109/ICRA.2013.6630752.

[31] Caetano, J. V., de Visser, C. C., Remes, B. D. W., de Wagter, C., and Mulder, M., "Controlled flight maneuvers of a Flapping Wing Micro Air Vehicle: a step towards the DelFly II Identification," AIAA Atmospheric Flight Mechanics Conference, No. 2013-4843, AIAA, 2013. doi:10.2514/6.2013-4843.

[32] de Croon, G. C. H. E., M. Perçin, B. D. W. R., Ruijsink, R., and De Wagter, C., The DelFly: Design, Aerodynamics, and Artificial Intelligence of a Flapping Wing Robot, Springer, 2015, pp. 31-54.

[33] Remes, B. D. W., Esden-Tempskiy, P., van Tienen, F., Smeur, E., De Wagter, C., and de Croon, G. C. H. E., "Lisa-S 2.8g autopilot for GPS-based flight of MAVs," International Micro Air Vehicle Conference and Competition (IMAV), Delft, The Netherlands, 2014, pp. 280-285. doi:10.4233/uuid:29e5367f-6c16-43e1-989e-d51cf8b51f7d.

[34] “Paparazzi UAV," [website], http: / / wiki.paparazziuav.org, [retrieved 7 Dec. 2016].

[35] Karásek, M., Koopmans, A. J., Armanini, S. F., Remes, B. D. W., and de Croon G. C. H. E., "Free Flight Force Estimation of a 23.5 g Flapping Wing MAV using an on-board IMU," International conference on intelligent robots and systems (IROS), Daejeon, Korea, 2016. doi:10.1109/IROS.2016.7759729.

[36] Gelb, A., editor, Applied Optimal Estimation, MIT Press, Cambridge, MA, 16th ed., 1974, pp. 180190.

[37] Zeyghami, S. and Dong, H., "Coupling of the wings and the body dynamics enhances damselfly maneuverability," ArXiv e-prints, Feb. 2015. 